\title{
A new SS-closedness in L-topological spaces
}

\author{
Shi-Zhong Bai \\ School of Mathematics and \\ computational science \\ Wuyi University \\ Guangdong, P.R.China \\ shizhongbai@yahoo.com.cn
}

\author{
Fang-Juan Zhang \\ School of Mathematics and \\ computational science \\ Wuyi University \\ Guangdong, P.R.China \\ zfj2008.love@163.com
}

\begin{abstract}
By means of strongly semi-open L-sets and their inequality, a new form of SS-closedness is introduced in $L$ - topological spaces, where $L$ is a complete De Morgan algebra. This new form does not depend on the structure of basis lattice $L$ and $L$ does not require any distributivity. When $L$ is a completely distributive De Morgan algebra, its many characterizations are presented.

Keywords-L-topology; strongly semi-open L -set; SSclosedness.
\end{abstract}

\section{INTRODUCTION}

As we know, compactness and its stronger and weaker forms play very important roles in general topology. In [1], we introduced the concepts of strongly semi-open sets and strongly semi-continuous mappings in $[0,1]$-topological spaces. Based on this, in [2], we introduced the concept of SR-compactness in L-topological spaces long Wang's [11] and Zhao's [13] compactness. In [3], we introduced the concept of SS-closedness in L-topological spaces along Kudri's compactness in [7].

However, Wang's compactness and Kudri's compactness, as well as the SR-compactness and the SS-closedness rely on the structure of $\mathrm{L}$ and $\$ \mathrm{~L} \$$ is required to be completely distributive. In [10], by means of open L-sets and their inequality, Shi introduced a new definition of fuzzy compactness in L-topological spaces, where $\mathrm{L}$ is a complete de Morgan algebra. This new definition doesn't depend on the structure of $L$. In [4], we introduced the concept of SRcompactness in $L$-topological spaces along Shi's compactness in [10].

In this paper, following the lines of [10], we'll introduce a new form of SS-closedness in $L$-topological spaces by means of strongly semi-open $L$-sets and their inequality. It is weaker form of SR-compactness. It can also be characterized by strongly semi-closed $L$-sets and their inequality. This new form of SS-closedness has many characterizations if $L$ is completely distributive De Morgan algebra.

\section{PRELIMINARIES}

Throughout this paper, $(\mathrm{L}, \vee, \wedge, ')$ is a complete De Morgan algebra, $\mathrm{X}$ a nonempty set. $L^{X}$ is the set of all Lfuzzy sets (or L-sets for short) on X. The smallest element and the largest element in $L^{X}$ are denoted by 0 and 1. An element $\mathrm{a}$ in $L$ is called prime element if $b \wedge c \leq a$ implies $b \leq a$ or $c \leq a$. a in $\mathrm{L}$ is called a co-prime element if $\mathrm{a}^{\prime}$ is a prime element [6]. The set of nonunit prime elements in $\mathrm{L}$ is denoted by $\mathrm{P}(\mathrm{L})$. The set of nonzero coprime elements in $\mathrm{L}$ is denoted by $\mathrm{M}(\mathrm{L})$.

The binary relation $\prec$ in $\mathrm{L}$ is defined as follows: or $a, b \in L, a \prec b$ iff for every subset $D \subseteq L$, the relation $b \leq \sup D$ always implies the existence of $d \in D$ with $a \leq d$ [5]. In a completely distributive De Morgan algebra L, each element $b$ is a sup of $\{a \in L \mid a \prec b\} .\{a \in L \mid a \prec b\}$ is called the greatest minimal family of $b$ in the sense of [8,12], in symbol $\beta(b)$. Moreover for $b \in L$, define $\beta^{*}(b)=\beta(b) \cap M(L) \quad, \quad \alpha(b)=\left\{a \in L \mid a^{\prime} \prec b^{\prime}\right\}$ and $. \alpha^{*}(b)=\alpha(b) \wedge P(L)$. For $a \in L$ and $A \in L^{X}$, we denote

$$
\begin{aligned}
& A^{(a)}=\{x \in X \mid A(x) \not a\} \quad, \quad A_{(a)}=\{x \in X \mid a \in \beta(A(x))\}, \\
& A^{[a]}=\{x \in X \mid a \notin \alpha(A(x))\} \text { and } A_{[a]}=\{x \in X \mid A(x) \geq a\} .
\end{aligned}
$$

For a subfamily $\psi \subseteq L^{X}, 2^{(\psi)}$ denotes the set of all finite subfamilies of $\psi$, and $2^{[\psi]}$ denotes the set of all countable subfamilies of $\psi$. An L-topological space denotes $L$-ts for short. Let $(X, \tau)$ be a weakly induce $L$-ts, $a \in L, A \in \tau$.Then $A_{(a)}$ is an open set in $[\tau]$, where $[\tau]$ is the topology formed by all crisp sets in $\tau$ [8].

Let $(X, \delta)$ be an $L$-ts and $A \in L^{X}$. Then $A$ is called a strongly semi-open set iff there is a $B \in \delta$ such that $B \leq A \leq B^{-o}$, and $A$ is called a strongly semi-closed set iff there is a $B \in \delta^{\prime}$ such that $B^{o-} \leq A \leq B[1,2]$, where $B^{o}$ and $B^{-}$are the interior and closure of $B$, respectively. The sets $A^{\Delta}=\vee\{B: B \quad$ is strongly semi-open, $B \leq A\} \quad$ and $A^{\sim}=\wedge\{B: B$ is strongly semi-closed, $B \geq A\}$ are called the strong semi-interior and the strong semi-closure of $A[1,2]$, respectively. 
Definition 2.1([4]) Let $(X, \delta)$ be an $L$-ts. $A \in L^{X}$ is called SR-compact if for every family $\mu$ of strongly semi-open $L$-sets, it follows that

$$
\wedge \underset{x \in X}{\wedge}\left(A^{\prime}(x) \vee \vee B(x)\right) \leq \underset{B \in \mu}{\vee}(\mu) \underset{x \in X}{\wedge}\left(A^{\prime}(x) \vee \underset{B \in V}{\vee} B(x)\right)
$$

Definition 2.2([9,10]) Let $(X, \delta)$ be an $L$-ts, $a \in L \backslash\{1\}$ and $A \in L^{X}$. A family $\mu \subseteq L^{X}$ is called

(1) an $a$-shading of $A$ if for any $x \in X$,

$$
\left(A^{\prime}(x) \vee \vee_{B \in \mu} B(x)\right) \nless \leq a .
$$

(2) a strong a-shading (briefly S-a-shading) of $\mathrm{A}$ if

$$
\wedge_{x \in X}\left(A^{\prime}(x) \vee \vee_{B \in \mu} B(x)\right) \not a .
$$

(3) an a-R-neighborhood family (briefly a-R-NF) of A if for any $x \in X,\left(A(x) \wedge \wedge_{B \in \mu} B(x)\right) \geq a$.

(4)a strong a-R-neighborhood family (briefly S-a-R-NF) of $\mathrm{A}$ if $\vee_{x \in X}\left(A(x) \wedge \wedge_{B \in \mu} B(x)\right) \geq a$.

Definition 2.3([9]) Let $(X, \delta)$ be an $L$-ts, $a \in L \backslash\{0\}$ and $A \in L^{X}$. A family $\mu \subseteq L^{X}$ is called

(1) a $\beta_{a}$-cover of $A$ if for any $x \in X$, it follows that

$$
a \in \beta\left(A^{\prime}(x) \vee \vee_{B \in \mu} B(x)\right) .
$$

(2) a strong $\beta_{a}$-cover (briefly S- $\beta_{a}$-cover) of $A$ if

$$
a \in \beta\left(\wedge_{x \in X}\left(A^{\prime}(x) \vee \vee_{B \in \mu} B(x)\right)\right)
$$

(3) a $Q_{a}$-cover of $A$ if for any $x \in X$, it follows that

$$
A^{\prime}(x) \vee \vee_{B \in \mu} B(x) \geq a .
$$

It is obvious that an S- $a$-shading of $A$ is an $a$-shading of $A$, that an S- $a$-R-NF of $A$ is an $a$-R-NF of $A$, that $\mu$ is an S- $a$-R-NF of $A$ iff $\mu^{\prime}$ is an S- $a$-shading of $A$, that an S- $\beta_{a}$-cover of $A$ must be a $\beta_{a}$-cover of $A$, and that a $\beta_{a}$-cover of $A$ must be a $Q_{a}$-cover of $A$.

Lemma 2.4 ([9]) Let $L$ be a complete Heyting algebra, $f: X \rightarrow Y$ be a map and $f_{L}: L^{X} \rightarrow L^{Y}$ is the extension of $f$, then for any family $\psi \subseteq L^{Y}$

$$
\underset{y \in Y}{\vee}\left(f_{L} \rightarrow(A)(y) \wedge \underset{B \in \psi}{\wedge} B(y)\right)=\underset{x \in X}{\vee}\left(A(x) \wedge \underset{B \in \psi}{\wedge} f_{L}^{\rightarrow} B(x)\right)
$$

Definition 2.5([2]) Let $(X, \delta)$ and $(Y, \tau)$ be two $L$-ts's. A map $f:(X, \delta) \rightarrow(Y, \tau)$ is called S-irresolute if $f_{L}^{\leftarrow}(B)$ is strongly semi-open in $(X, \delta)$ for every strongly semi-open $L$ set $B$ in $(Y, \tau)$.

Theorem 2.6 ([2]) A map $f:(X, \delta) \rightarrow(Y, \tau)$ is S-irresolute iff $f_{L}^{\leftarrow}\left(B^{\Delta}\right) \leq\left(f_{L}(B)\right)^{\Delta}$ for each $B \in L^{Y}$.

\section{DEFINITION AND CHARACTERIZATIONS OF SS-}

\section{CLOSEDNESS}

Definition 3.1 Let $(X, \delta)$ be an $L$-ts. $A \in L^{X}$ is called SS-closed if for every family $\mu$ of strongly semi-open $L$ sets, it follows that

$$
\wedge \hat{x \in X}\left(A^{\prime}(x) \vee \underset{B \in \mu}{\vee} B(x)\right) \leq \underset{v \in 2}{\vee}(\mu) \underset{x \in X}{\wedge}\left(A^{\prime}(x) \vee \underset{B \in \mu}{\vee} B^{\sim}(x)\right) .
$$

Obviously we have the following result.

$$
\text { SR-compactness } \Rightarrow \text { SS-closedness. }
$$

That the converse of above need not be true is shown by the Example 3.2.

Example 3.2 Let $X$ be an infinite set(or $X$ be a singleton); $A$ and $C$ be two $[0,1]$-sets on $\mathrm{X}$ defined as $\mathrm{A}(\mathrm{x})=0.6$, for all $x \in X ; \mathrm{C}(\mathrm{x})=0.8$, for all $x \in X$. Take $\delta=\{\phi, A, X\}$, then $\delta$ is a topology on $X$. We easily obtain that $C$ is SS-closed. But $C$ is not SR-compact.

From the Definition 3.1, we can obtain the following theorem by using quasi-complement.

Theorem 3.3 Let $(X, \delta)$ be an $L$-ts. $A \in L^{X}$ is SS-closed iff for every family $\mu$ of strongly semi-closed L-sets, it follows that

$$
\underset{x \in X}{\vee}(A(x) \wedge \underset{B \in \mu}{\wedge} B(x)) \geq \underset{v \in 2}{\wedge}(\mu) \underset{x \in X}{\vee}\left(A(x) \wedge \underset{B \in \nu}{\wedge} B^{\Delta}(x)\right) .
$$

From the Definition 3.1 and the Theorem 3.4, we immediately obtain the following the result.

Theorem 3.4 Let $(X, \delta)$ be an $L$-ts and $A \in L^{X}$. Then the following conditions are equivalent. 
(1) $A$ is SS-closed.

(2) For any $\mathrm{a} \in \mathrm{L} \backslash\{1\}$, each strongly semi-open S- $a$ shading $\mu$ of $A$ has a finite subfamily $v$ such that $\left\{B^{\sim} \mid B \in v\right\}$ is an S- $a$-shading of $A$.

(3)For any $a \in L \backslash\{0\}$, each strongly semi-closed S- $a$-R$\mathrm{NF} \psi$ of $A$ has a finite subfamily $\varphi$ such that $\left\{B^{\Delta} \mid B \in \varphi\right\}$ is an S- $a$-R-NF of $A$.

Theorem 3.5 If $C$ is SS-closed and $D$ is strongly semiclosed, then $C \wedge D$ is SS-closed.

Proof. For any family $\mu$ of strongly semi-closed $L$-sets, by the Theorem 3.4 we have that

$$
\begin{aligned}
& \underset{x \in X}{\vee}((C \wedge D)(x) \wedge \underset{B \in \mu}{\wedge} B(x))=\underset{x \in X}{\vee}(C(x) \wedge \underset{B \in \mu \cup\{D\}}{\wedge} B(x)) \\
& \geq \underset{v \in 2}{ }(\hat{\mu \cup\{D\}})_{x \in X}^{\vee}\left(C(x) \wedge \underset{B \in V}{\wedge} B^{\Delta}(x)\right) \\
& =\left\{\underset{v \in 2}{\wedge}(\mu) \underset{x \in X}{\vee}\left(C(x) \wedge \underset{B \in V}{\wedge} B^{\Delta}(x)\right)\right\} \\
& \wedge\left\{\underset{v \in 2}{\wedge}(\mu) \underset{x \in X}{\vee}\left(C(x) \wedge D(x) \wedge \underset{B \in V}{\wedge} B^{\Delta}(x)\right)\right\} \\
& \left\{\underset{v \in 2}{\wedge}(\mu) \underset{x \in X}{\vee}\left(C(x) \wedge D(x) \wedge \underset{B \in v}{\wedge} B^{\Delta}(x)\right)\right\}
\end{aligned}
$$

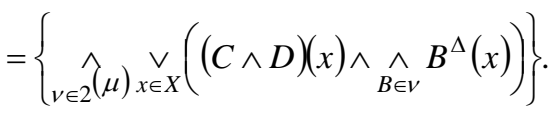

This shows that $C \wedge D$ is SS-closed.

Theorem 3.6 Let $L$ be a complete Heyting algebra. If both $C$ and $D$ are SS-closed, then $C \vee D$ is SS-closed.

Proof. For any family $\mu$ of strongly semi-closed $L$-sets, by the Theorem 3.4 we have that

$$
\begin{aligned}
& \underset{x \in X}{\vee}((C \vee D)(x) \wedge \underset{B \in \mu}{\wedge} B(x)) \\
& =\{\underset{x \in X}{\vee}(C(x) \wedge \underset{B \in \mu}{\wedge} B(x))\} \vee\{\underset{x \in X}{\vee}(D(x) \wedge \underset{B \in \mu}{\wedge} B(x))\} \\
& \geq\left\{\underset{v \in 2}{\wedge}(\mu) \underset{x \in X}{\vee}\left(C(x) \wedge \underset{B \in V}{\wedge} B^{\Delta}(x)\right)\right\} \\
& \vee\left\{\underset{v \in 2}{\wedge}(\mu) \underset{x \in X}{\vee}\left(D(x) \wedge \underset{B \in V}{\wedge} B^{\Delta}(x)\right)\right\} \\
& =\underset{v \in 2}{\wedge}(\mu) \underset{x \in X}{\vee}\left((C \vee D)(x) \wedge \underset{B \in V}{\wedge} B^{\Delta}(x)\right)
\end{aligned}
$$

This shows that $C \vee D$ is SS-closed.

Theorem 3.7 Let $L$ be a complete Heyting algebra and $f:(X, \delta) \rightarrow(Y, \tau)$ be an S-irresolute map. If $A$ is an SSclosed $L$-set in $(X, \delta)$, then so is $f_{L} \rightarrow(A)$ in $(Y, \tau)$.

Proof. Suppose that $\mu$ is a family of strongly semi-closed $L$-sets in $(Y, \tau)$, by the Lemma 2.4, the Theorem 2.6 and SS-closed of $A$, we have that

$$
\begin{aligned}
& \underset{y \in Y}{\vee}\left(f_{L}^{\rightarrow}(A)(y) \wedge \underset{B \in \mu}{\wedge} B(y)\right) \\
& =\underset{x \in X}{\vee}\left(A(x) \wedge \underset{B \in \mu}{\wedge} f_{L}^{\leftarrow} B(x)\right) \\
& \geq \underset{v \in 2}{ }(\mu) \underset{x \in X}{\vee}\left(A(x) \wedge \underset{B \in \nu}{\wedge}\left(f_{L}^{\leftarrow}(B)\right)^{\Delta}(x)\right) \\
& \geq \underset{v \in 2}{\wedge}(\mu) \underset{x \in X}{\vee}\left(A(x) \wedge \underset{B \in V}{\wedge} f_{L}^{\leftarrow}\left(B^{\Delta}\right)(x)\right) \\
& =\underset{v \in 2}{\hat{2}} \underset{(\mu)}{\vee} \underset{y \in Y}{\vee}\left(f_{L} \rightarrow(A)(y) \wedge \underset{B \in V}{\wedge} B^{\Delta}(y)\right) .
\end{aligned}
$$

Therefore $f_{L} \rightarrow(A)$ is SS-closed.

\section{FURTHER PROPERTIES OF SS-CLOSEDNESS}

In this section, we assume that $L$ is a completely distributive de Morgan algebra.

Theorem 4.1 Let $(X, \delta)$ be an $L$-ts and $A \in L^{X}$.

Then the following conditions are equivalent.

(1) $A$ is SS-closed.

(2) For any $a \in L \backslash\{0\}$, each strongly semi-closed S- $a$ R-NF $\psi$ of $A$ has a finite subfamily $\varphi$ such that $\left\{B^{\Delta} \mid B \in \varphi\right\}$ is an $a-\mathrm{R}-\mathrm{NF}(\mathrm{S}-a-\mathrm{R}-\mathrm{NF})$ of $A$. 
(3) For any $a \in L \backslash\{0\}$ and any strongly semi-closed S$a-\mathrm{R}-\mathrm{NF} \psi$ of $\$ \mathrm{~A} \$$, there is a finite subfamily $\varphi$ of $\psi$ and $b \in \beta(a)$ such that $\left\{B^{\Delta} \mid B \in \varphi\right\}$ is a $b$-R-NF (an S- $b$-RNF) of $A$.

(4) For any $a \in L \backslash\{1\}$, each strongly semi-open S- $a$ shading $\mu$ of $A$ has a finite subfamily $v$ such that $\left\{B^{\sim} \mid B \in v\right\}$ is an ( $a$-shading) S- $a$-shading of $A$.

(5) For any $a \in L \backslash\{1\}$ and any strongly semi-open S- $a$ shading $\mu$ of $A$, there is a finite subfamily $v$ of $\mu$ and $b \in \alpha(a)$ such that $\left\{B^{\sim} \mid B \in v\right\}$ is a $b$-shading (an S- $b$ shading) of $A$.

(6) For any $a \in L \backslash\{0\}$, each strongly semi-open S- $\beta_{a}$ cover $\mu$ of $A$ has a finite subfamily $v$ such that $\left\{B^{\sim} \mid B \in v\right\}$ is a $\beta_{a}$-cover (an S- $\beta_{a}$-cover) of $A$.

(7) For any $a \in L \backslash\{0\}$ and any strongly semi-open S$\beta_{a}$-cover $\mu$ of $A$, there is a finite subfamily $v$ of $\mu$ and $b \in L$ with $a \in \beta(b)$ such that $\left\{B^{\sim} \mid B \in v\right\}$ is a $\beta_{a}$-cover (an S- $\beta_{a}$-cover) of $A$.

(8)For any $a \in L \backslash\{0\}$ and any $b \in \beta(a) \backslash\{0\}$, each strongly semi-open $Q_{a}$-cover $\mu$ of $A$ has a finite subfamily $v$ of $\mu$ such that $\left\{B^{\sim} \mid B \in v\right\}$ is a $Q_{b}$-cover of $A$.

(9) For any $a \in L \backslash\{0\}$ and any $b \in \beta(a) \backslash\{0\}$, each strongly semi-open $Q_{a}$-cover $\mu$ of $A$ has a finite subfamily $v$ of $\mu$ such that $\left\{B^{\sim} \mid B \in v\right\}$ is a $\beta_{b}$-cover (an S- $\beta_{b}$-cover) of $A$.

Proof. This is immediate from the Definition 3.1 and the

Theorem 3.5.

Lemma 4.2([4]). Let $\left(X, \omega_{L}(\tau)\right)$ be generated topologically by $(X, \tau)$. If $A$ is a strongly semi-open $L$-set in $(X, \tau)$, then $\chi_{A}$ is a strongly semi-open set in $\left(X, \omega_{L}(\tau)\right)$. If $B$ is a strongly semi-open $L$-set in $\left(X, \omega_{L}(\tau)\right)$, then $B_{(a)}$ is a strongly semi-open set in $(X, \tau)$ for every $a \in L$.

Since $B^{\sim}$ is the smallest strongly semi-closed $L$-set which contains $B$ and $B^{\Delta}$ is the greatest strongly semiopen $L$-set which is contained in $B$, we can obtain the following lemma.
Lemma 4.3. Let $\left(X, \omega_{L}(\tau)\right)$ be generated topologically by $(X, \tau)$. Then $\left(B_{[b]}\right)^{\sim} \subseteq\left(B^{\sim}\right)_{[b]}$ for any $b \in L$ for any $B \in L^{X}$, where $\left(B_{[b]}\right)^{\sim}$ and $B^{\sim}$ denote the strongly semiclosures of $B_{[b]}$ and $B$ in $(X, \tau)$ and $\left(X, \omega_{L}(\tau)\right)$ respectively.

Theorem 4.4. Let $(X, \tau)$ be a topological space and $\left(X, \omega_{L}(\tau)\right)$ be generated topologically by $(X, \tau)$. Then $\left(X, \omega_{L}(\tau)\right)$ is SS-closed iff $(X, \tau)$ is SS-closed.

Proof. Necessity. Let $\mu$ be a strongly semi-open cover of $(X, \tau)$. Then $\left\{\chi_{A} \mid A \in \mu\right\}$ is a family of strongly

semi-open $\quad L \quad$-sets $\quad$ in $\quad\left(X, \omega_{L}(\tau)\right) \quad$ with $\wedge_{x \in X}\left(\vee_{A \in \mu} \chi_{A}(x)\right)=1$.

From SS-closedness of $\left(X, \omega_{L}(\tau)\right)$, we have that

$$
\underset{v \in 2}{\vee}(\mu) \underset{x \in X}{\wedge}\left(\underset{A \in V}{\vee} \chi_{A^{\sim}}(x)\right) \geq \underset{v \in 2}{\vee}(\mu) \underset{x \in X}{\wedge}\left(\underset{A \in V}{\vee}\left(\chi_{A}\right)^{\sim}(x)\right)=1 .
$$

This implies that there exists $v \in 2^{(\mu)}$ such that

$$
\wedge_{x \in X}\left(\vee_{A \in V} \chi_{A^{\sim}}(x)\right)=1 .
$$

Hence, $\left\{A^{\sim} \mid A \in v\right\}$ is a cover of $(X, \tau)$. Therefore $(X, \tau)$ is SS-closed.

Sufficiency. Let $\mu$ be a family of strongly semi-open $L$ sets in $\left(X, \omega_{L}(\tau)\right)$ and $\wedge_{x \in X}\left(\vee_{B \in \mu} B(x)\right)=a$.

If $a=0$, obviously we have that

$$
\wedge \underset{x \in X}{\wedge} \underset{B \in \mu}{\vee} B(x)) \leq \underset{v \in 2}{\vee}(\mu) \underset{x \in X}{\wedge}\left(\underset{B \in \nu}{\vee} B^{\sim}(x)\right) .
$$

Now we suppose that $a \neq 0$. In this case, for any $b \in \beta(a) \backslash\{0\}$, we have that

$$
b \in \beta(\underset{x \in X}{\wedge}(\underset{B \in \mu}{\vee} B(x))) \subseteq \bigcap_{x \in X} \beta(\underset{B \in \mu}{\vee} B(x))=\bigcap_{x \in X} \bigcup_{B \in \mu} \beta(B(x))
$$

From the Lemma 4.2, this implies that $\left\{B_{(b)} \mid B \in \mu\right\}$ is a strongly semi-open cover of $(X, \tau)$. From SS-closedness 
of $(X, \tau)$, we know that there exists $v \in 2^{(\mu)}$ such that $\left\{\left(B_{(b)}\right) \sim \mid B \in v\right\}$ is a cover of $(X, \tau)$. Obviously $\left\{\left(B^{\sim}\right)_{[b]} \mid B \in v\right\} \quad$ is a cover of $(X, \tau)$ since $\left(B_{(b)}\right) \subseteq\left(B_{[b]}\right) \sim\left(B^{\sim}\right)_{[b]}$. Hence $b \leq \wedge_{x \in X}\left(\vee_{B \in V} B^{\sim}(x)\right)$

Further, we have that

$$
b \leq \underset{x \in X}{\wedge}\left(\underset{B \in V}{\vee} B^{\sim}(x)\right) \leq \underset{v \in 2}{\vee}(\mu) \underset{x \in X}{\wedge}\left(\underset{B \in V}{\vee} B^{\sim}(x)\right) .
$$

This implies that

$$
\underset{x \in X}{\wedge}(\underset{B \in \mu}{\vee} B(x))=a=\vee\{b \mid b \in \beta(a)\} \leq \underset{v \in 2}{\vee}(\mu) \underset{x \in X}{\wedge}\left(\underset{B \in v}{\vee} B^{\sim}(x)\right)
$$

Therefore, $\left(X, \omega_{L}(\tau)\right)$ is SS-closed.

\section{ACKNOWLEDGMENT}

This work is supported by the NSFC of China (No. 61070150), the NSF of Guangdong Province (No. S2012010008833), the Project of DEGP (No. 2012KJCX0101) and STF of Jiangmen City (No. 2012-156).

\section{REFERENCES}

[1] S.Z. Bai, " Fuzzy strongly semiopen sets and fuzzy strong semicontinuity,"Fuzzy Sets and Systems, 52(1992) 345-351.

[2] S.Z. Bai, "The SR-compactness in $L$-fuzzy topological spaces,"Fuzzy Sets and Systems, 87(1997) 219-225.

[3] S.Z.Bai, "SS-closedness in $L$-topological spaces,"Indian J.Pure Appl.Math, 34(2003) 1697-1706.

[4] S.Z. Bai, "A new form of SR-compactness in $L$-topological Spaces,"Fuzzy Systems and Mathematics, 21(2007) 57-62 .

[5] P. Dwinger, "Characterizations of the complete homomorphic images of a completely distributive complete lattice,"I, Nederl. Akad. Wetensch. Indag. Math, 44(1982) 403-414.

[6] G. Gierz, et al, “,A Compendium of Continuous Lattices,”Springer Verlag, Berlin, 1980.

[7] S.R.T.Kudri, Compactness in $L$-fuzzy topological spaces“,"Fuzzy Sets and Systems, 67(1994) 329-336 .

[8] Y.M. Liu and M.K. Luo, "Fuzzy Topology,World Scienti.c, Singapore, 1998".

[9] F.G.Shi, "A new notion of fuzzy compactness in $L$-topological spaces,"Information Sciences, 173(2005) 35-48.

[10] F.G.Shi, "A new definition of fuzzy compactness,"Fuzzy Sets and Systems, 158(13)(2007) 1486-1495.

[11] G.J.Wang, A new fuzzy compactness defined by fuzzy nets“,"J.Math.Anal.Appl, 94(1983) 1-23.

[12] G.J.Wang, "Theory of L-Fuzzy Topological Space," .Shaanxi Normal University Press, Xian, 1988.

[13] D.S.Zhao, "The N-compactness in $L$-fuzzy topological spaces,”J.Math.Anal.Appl, 128(1987) 64-79 